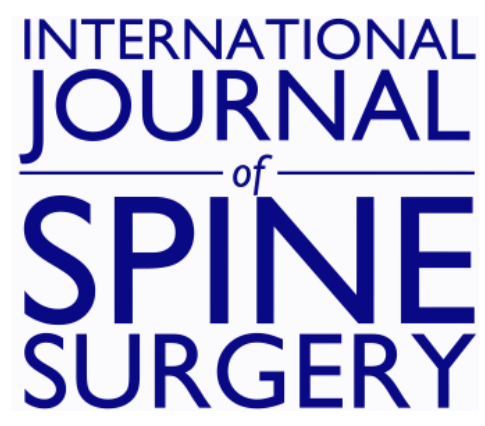

\title{
Radiologic Analysis of C2 to Predict Safe Placement of Pedicle Screws
}

REX A.W. MARCO, CHRISTOPHER I. PHELPS, REBECCA C. KUO, WU ZHUGE, CLINTON W. HOWARD, VIVEK P. KUSHWAHA and DEREK T. BERNSTEIN

Int J Spine Surg 2018, 12 (1) 30-36

doi: https://doi.org/10.14444/5006

http://ijssurgery.com/content/12/1/30

This information is current as of April 26, 2023.

Email Alerts Receive free email-alerts when new articles cite this article. Sign up at:

http://ijssurgery.com/alerts

The International Journal of Spine Surgery

2397 Waterbury Circle, Suite 1,

Aurora, IL 60504, Phone: +1-630-375-1432 


\title{
Radiologic Analysis of C2 to Predict Safe Placement of Pedicle Screws
}

\author{
REX A.W. MARCO, MD, ${ }^{1,2}$ CHRISTOPHER I. PHELPS, MD, ${ }^{2}$ REBECCA C. KUO, MD,${ }^{2}$ WU ZHUGE, MD, ${ }^{2}$ \\ CLINTON W. HOWARD, MD, ${ }^{2}$ VIVEK P. KUSHWAHA, MD, ${ }^{2}$ DEREK T. BERNSTEIN, MD ${ }^{1}$ \\ ${ }^{1}$ Houston Methodist Hospital, Department of Orthopaedic Surgery, Houston, Texas, ${ }^{2}$ University of Texas Medical School at Houston, Department of Orthopaedic \\ Surgery, Houston, Texas
}

\begin{abstract}
Background: Preoperative assessment of $\mathrm{C} 2$ pedicle morphology is critical to safe pedicle screw placement. To avoid iatrogenic injury, complex digital templating software has been introduced; however, this technology may not be available in many centers. We report a technique for preoperative assessment of C2 pedicle screw placement safety based upon 2-dimensional sagittal computed tomography (CT) scan images and verify its utility in clinical practice.

Methods: A total of 46 consecutive patients underwent cervical spine CT scans between 2005 and 2011. The C2 pedicle morphology was assessed on sagittal CT imaging by 5 independent reviewers to determine the feasibility and risk associated with pedicle screw placement. Thirty consecutive patients underwent $\mathrm{C} 2$ pedicle screw placement and were followed clinically for a minimum of 2 years. The ability to place a screw was noted, and accuracy of screw placement was assessed postoperatively by CT scan.

Results: The CT scan analysis demonstrated that 11\% (5/46) of patients had sufficient pedicle size bilaterally to allow safe placement of long pedicle screws with a low risk of vertebral artery injury, whereas $15 \%(7 / 46)$ were considered a high risk bilaterally. Screw placement was deemed low risk in $28 \%$, moderate risk in $38 \%$, and high risk in $34 \%$. Excellent intraobserver reliability and good interobserver reliability was observed. Clinically, 18 of $20(90 \%)$ low-risk and 21 of $24(88 \%)$ moderate-risk pedicle screws were placed safely versus 5 of $16(31 \%)$ high-risk pedicle screws $(P<.001)$.

Conclusions: Using the described technique for evaluating the $\mathrm{C} 2$ pedicle via sagittal CT scan images allows for safe and reliable pedicle screw placement without relying upon complex digital templating software, which may have limited availability.

Level of Evidence: II

Clinical Relevance: This study aids in the surgical decision-making behind the placement of $\mathrm{C} 2$ pedicle screws using CT scans without reliance upon complex digital templating software.
\end{abstract}

TDR

Keywords: pedicle screw, vertebral artery, CT scan, vertebral artery injury, C2 pedicle, high-riding vertebral artery

\section{INTRODUCTION}

Multiple techniques of posterior surgical fixation at $\mathrm{C} 2$, primarily for $\mathrm{C} 1-\mathrm{C} 2$ fusion, have been described, ${ }^{1}$ including sublaminar wiring, transarticular screw fixation, and more recently, the combination of $\mathrm{C} 1$ lateral mass and $\mathrm{C} 2$ pedicle screws connected by rods. ${ }^{2-5}$ This latter technique is particularly advantageous because it creates a more rigid $\mathrm{C} 1-\mathrm{C} 2$ construct compared with sublaminar wiring. Moreover, a C1-C2 transarticular screw cannot be safely placed in up to $20 \%$ of patients owing to the variability in $\mathrm{C} 2$ pedicle and vertebral artery foramen anatomy, with the most common anomaly being the so-called high-riding vertebral artery. $^{6-10}$
Whereas the vertebral artery remains at risk for injury during $\mathrm{C} 2$ pedicle screw placement, the more superomedial trajectory of this technique decreases this risk compared with $\mathrm{C} 1-\mathrm{C} 2$ transarticular screw placement. ${ }^{5}$ Nonetheless, preoperative assessment of the pedicle to evaluate for anatomic variation is important when planning for pedicle screw placement; however, currently reported techniques require the use of complex digital templating software with 3-dimensional computed tomography (CT) scan reconstructions, which may not be commonly available. $^{6,7,11,12}$

Therefore, the purpose of this study is to describe a more accessible technique for evaluating $\mathrm{C} 2$ pedicle anatomy relative to the vertebral artery using only sagittal CT imaging without the need for additional 


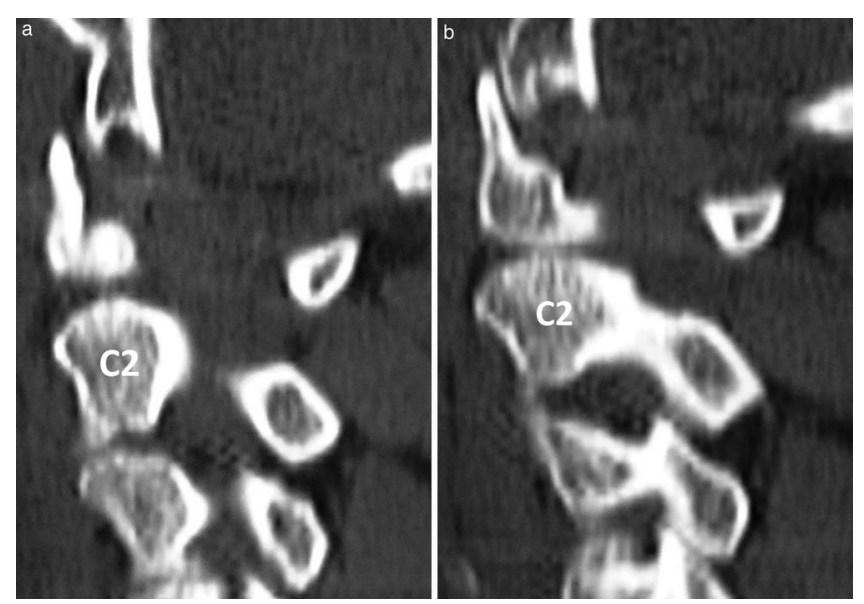

Figure 1. (a) Identification of the medial wall slice. Sagittal computed tomography reconstruction cut showing the lateral-most aspect of the spinal canal. (b) The next cut is $2 \mathrm{~mm}$ lateral to the lateral aspect of the spinal canal and shows the medial wall slice with complete continuity of the pedicle from the vertebral body to the posterior elements.

3-dimensional reconstruction or templating software. Intraobserver and interobserver reliability as well as subsequent clinical application are reported to determine efficacy and safety. This technique is simple and reproducible and should provide surgeons with the information required to predict the relative safety of $\mathrm{C} 2$ pedicle screw placement.

\section{MATERIALS AND METHODS}

Following institutional review board approval, a retrospective observational imaging study was performed using 46 cervical spine CT scan studies to define the theoretical risk associated with placement of $\mathrm{C} 2$ pedicle screws based upon the width of the bony corridor. Standard 2-mm sagittal CT scan images through the $\mathrm{C} 2$ vertebra were evaluated.

\section{Evaluation of Vertebral Artery Injury Risk in C2 Pedicle Screw Placement}

The theoretical safety of $\mathrm{C} 2$ pedicle screw placement was evaluated relative to the spinal canal and vertebral artery for each CT scan. The lateralmost aspect of the spinal canal on the left and right sides were identified individually. The medial wall of the pedicle was then identified as the first sagittal slice demonstrating complete bony continuity between the vertebral body and the lamina (Figure 1a and $b)$. This cut was labeled slice 1 .

The total number of sequential 2-mm slices from the medial wall of the pedicle up to, but not including, the vertebral artery foramen cut was then recorded. The first sagittal slice showing at least a

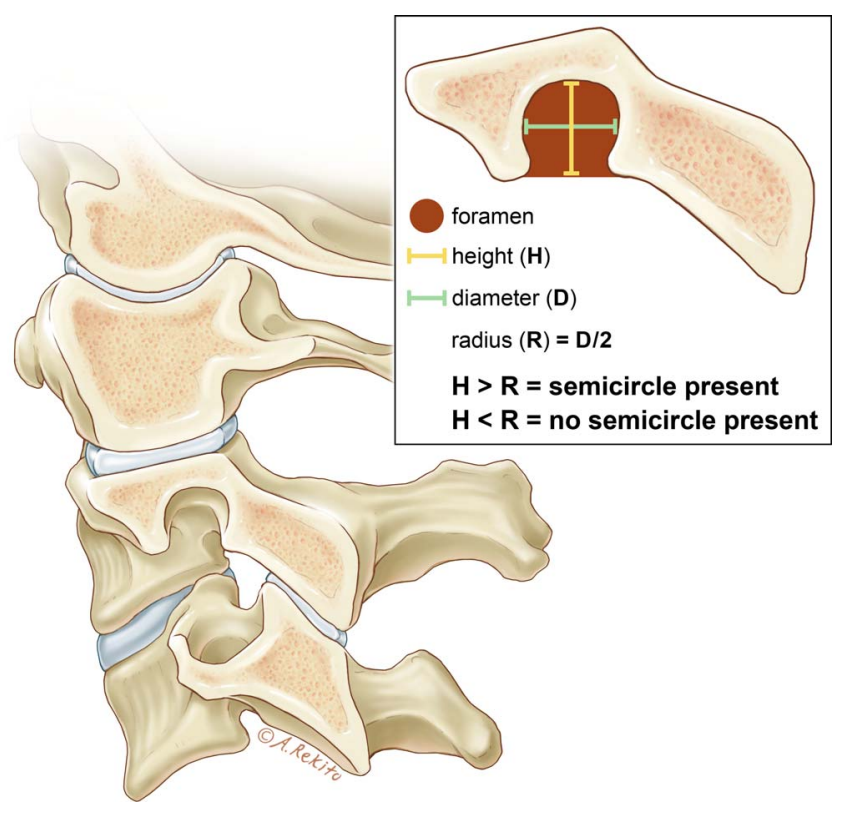

Figure 2. Identification of the vertebral artery foramen cut. The first cut that shows a complete semicircular opening is deemed the vertebral artery foramen cut. A complete semicircle is considered present when the height $(H)$ is greater than the radius $(R)$ of the foramen.

semicircular opening of the foramen (ie, an opening with a height greater than or equal to its radius) was considered the vertebral artery foramen cut (Figures 2, 3a and $b$ ).

Placement of a $3.5-$ or $4.0-\mathrm{mm}$ screw was considered at low risk of injuring the vertebral artery when at least 3 slices (ie, $\geq 4 \mathrm{~mm}$ of pedicle width) lateral to the canal did not show the vertebral artery foramen cut (Figure 4). Pedicles with 2 slices showing bony continuity were considered at moderate risk of injury, and those with 1 or no slices
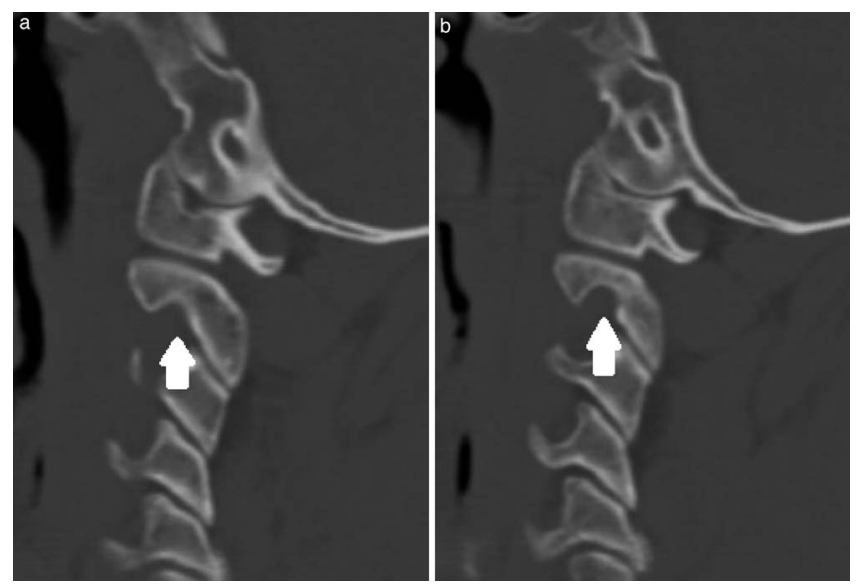

Figure 3. (a) Identification of the vertebral artery foramen slice. A small portion of the superomedial aspect of the vertebral artery foramen can be seen in the $\mathrm{C} 2$ pedicle. (b) The next cut is $2 \mathrm{~mm}$ lateral and demonstrates a vertebral artery foramen with at least a full semicircular opening. This cut is deemed the vertebral artery foramen slice. 


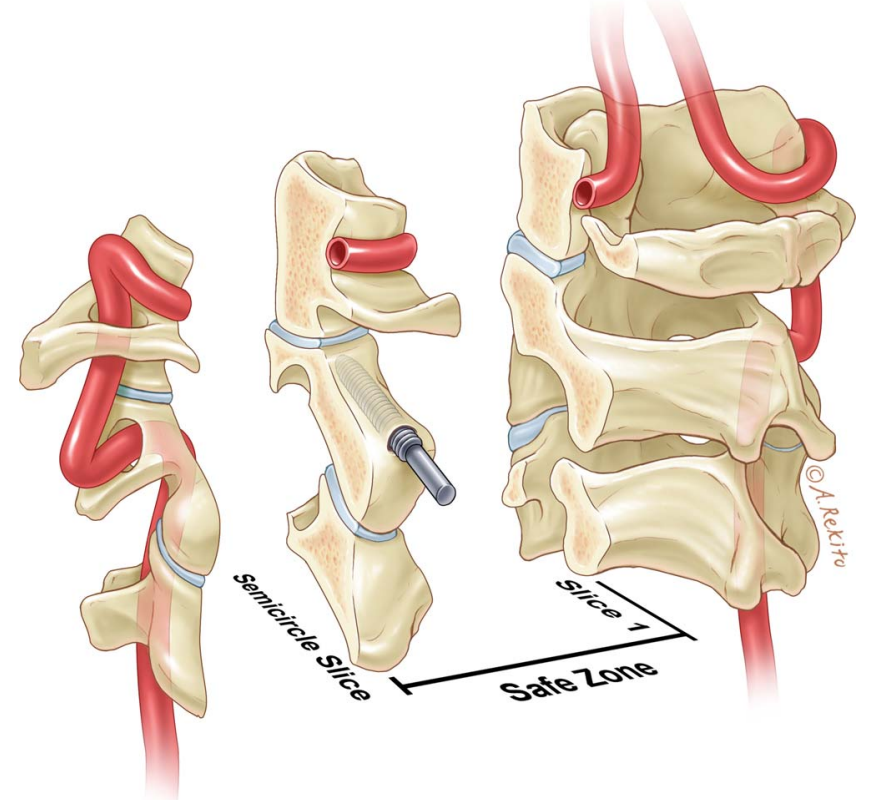

Figure 4. Safe zone for $\mathrm{C} 2$ pedicle screw placement between slice 1 and the semicircle slice.

showing bony continuity were considered at high risk of vertebral artery injury. Figure 5 shows the typical findings in a patient at low risk of vertebral artery injury. In this patient, there were at least 4 slices from the medial wall slice before the slice with the semicircle denoting the foramen was visualized.

To determine intraobserver and interobserver reliability of this technique, 5 reviewers, including 2 fellowship-trained spine surgeons, 2 spine surgery fellows, and 1 orthopedic surgery resident, evaluated the cervical spine CT scan studies of 46 consecutive skeletally mature patients identified in the orthopedic trauma registry at a single institution. Each reviewer was first given a slideshow tutorial detailing the technique for CT scan assessment. The reviewers were then instructed to count the number of slices beginning from the medial pedicle wall up to but not including the vertebral artery foramen cut (ie, the slice with the semicircular opening). Each reviewer performed this twice in a blinded fashion, unaware of his or her performance. This protocol was repeated for both the left and the right pedicle of each study.

\section{Statistical Methods}

The intraclass correlation coefficient (ICC) was used to determine both the interreviewer and intrareviewer reliability. Because both patient films and reviewers were viewed as random samples, the 2-way random model was used for this determination. Absolute agreement among physicians would show the complete reliability of this method, with the ICC reflecting less reliability depending on discrepancies in the counts. The unit of analysis was a single reviewer's scoring (ie, count) of a patient's CT scan.

The interobserver correlation coefficient was calculated for each reviewer using the data recorded for the left and right pedicles at the 2 different

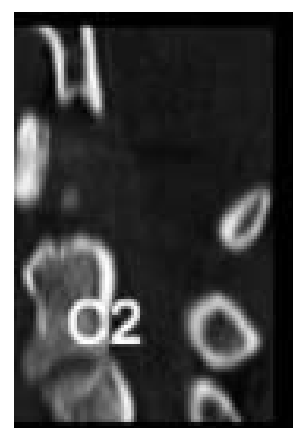

Canal

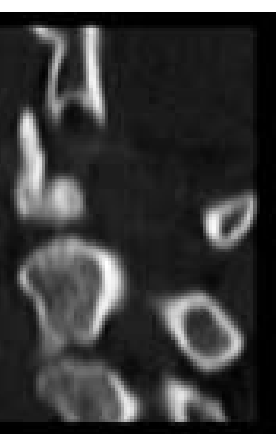

CanalNo bony continuity

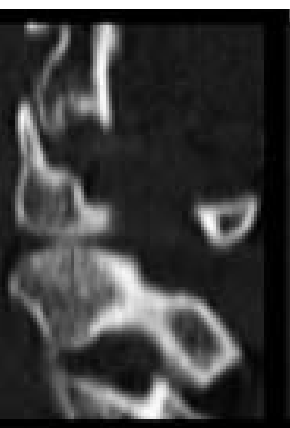

Slice 1-

Bony continuity

No semicircle of foramen

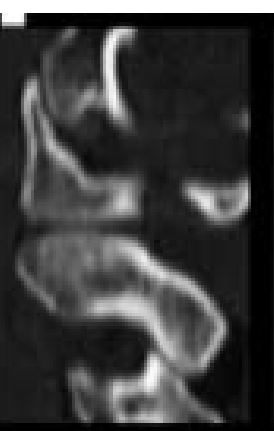

Slice 2-

No semicircle

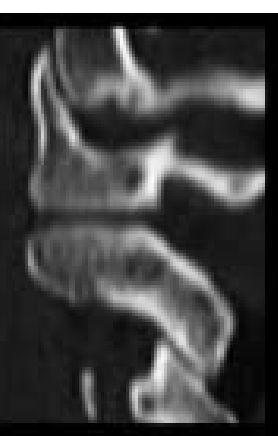

Slice 3No semicircle

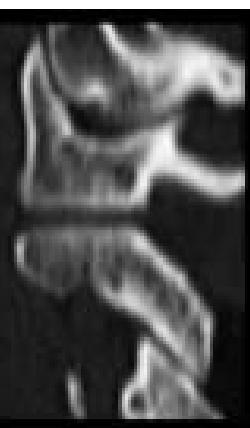

Slice 4$<$ semicircle of foramen

\section{.}


scorings. The intraobserver correlation coefficient was calculated for the group using the raw data recorded by each reviewer.

Outcomes evaluations were based on accepted standards for the interpretation of ICCs set forth by Fleiss and Cohen. ${ }^{13}$

$$
\begin{aligned}
& \text { - }<.4, \text { poor } \\
& \text { - } .4 \text { to } .75, \text { good } \\
& \text { - >.75, excellent }
\end{aligned}
$$

\section{Clinical Evaluation}

The feasibility and safety of placing $\mathrm{C} 2$ pedicle screws guided by preoperative sagittal CT scan evaluation as described above was assessed by the senior author in 30 consecutive patients who underwent instrumentation of the upper cervical spine between 2005 and 2011 with a 2-year minimum follow up. These 30 patients were separate from the cohort analyzed in the aforementioned observational imaging study. Each medical record was reviewed for demographic information, indications for surgery, implant and operative variables, and follow-up duration. Fifteen patients were male and 15 were female, with an average age of 54 years (range, 6 to 87 years). Pedicle screw placement was attempted on the basis of the clinical judgment of the operating surgeon, which took into account the combination of aberrant pedicle morphology, pedicle height less than $3.5 \mathrm{~mm}$, and 0 to 1 sagittal pedicle slices on preoperative CT. For moderateand high-risk pedicle screws, a more medial and cephalad starting point was used with increased superomedial angulation compared with low-risk pedicle screws. Complications associated with vertebral artery injury were recorded. Postoperative axial and sagittal CT scan images were obtained in $22(73 \%)$ patients, from which the incidence of cortical disruption into the spinal canal or vertebral artery foramen was determined. The Pearson $\chi^{2}$ test and the Fisher exact test were used to analyze categorical variables.

\section{Pedicle Screw Insertion Technique}

The medial wall of the pedicle is identified as a landmark with an angled ball-tipped probe. Subsequently, a burr is used to breach the posterior cortex approximately $2 \mathrm{~mm}$ lateral to the medial wall of the pedicle and $3 \mathrm{~mm}$ cephalad to the $\mathrm{C} 2-\mathrm{C} 3$ facet. A straight ball-tipped probe was then inserted at approximately $20^{\circ}$ medial angulation and $30^{\circ}$
Table 1. Comparison of $\mathrm{C} 2$ anatomic variation based on sagittal computed tomography cuts assuming placement of a long pedicle screw.

\begin{tabular}{lcc}
\hline Analysis & $\begin{array}{c}\text { No. of Pedicals at Risk/ } \\
\text { Total No. of Pedicles }\end{array}$ & $\mathbf{\%}$ \\
\hline Low risk bilaterally & $5 / 46$ & 11 \\
Moderate risk bilaterally & $9 / 46$ & 20 \\
High risk bilaterally & $7 / 46$ & 15 \\
Low risk/moderate risk & $8 / 46$ & 17 \\
Low risk/high risk & $8 / 46$ & 17 \\
Moderate risk/high risk & $9 / 46$ & 20 \\
Total low risk & $26 / 92$ & 28 \\
Total moderate & $35 / 92$ & 38 \\
Total high risk & $31 / 92$ & 34 \\
\hline
\end{tabular}

cephalad angulation with proper trajectory within the pedicle confirmed by lateral fluoroscopy. A battery-powered drill with a soft tissue protector preset to drill up to $16 \mathrm{~mm}$ for pars screws and 20 to $26 \mathrm{~mm}$ for pedicle screws was then used to intermittently advance the 2-mm drill, ensuring a proper cephalad trajectory with lateral fluoroscopy. Each pulse of the trigger advances the drill bit 1 to 2 $\mathrm{mm}$ whenever the drill bit is located within cancellous bone; however, if the drill bit stops advancing with each pulse of the trigger, then the tip of the drill bit may be abutting the cortical bone of the transverse foramen or spinal canal. In that case, the trajectory of the drill bit should be adjusted by directly palpating the medial wall of the pedicle and ensuring that the drill bit is angled slightly medial to avoid the vertebral artery but still lateral to the medial wall of the pedicle. Alternatively, pedicle screw placement could be aborted or a shorter pars screw could be placed. A ball-tipped probe is then used to palpate all 4 walls and the anterior cortex of the drill hole prior to placing the screw.

\section{RESULTS}

In the observational imaging portion of the study, $28 \%(26 / 92)$ of pedicles were deemed at low risk of injury to the vertebral artery. There was a moderate risk of vertebral artery injury in 38\% (35/92) and a high risk of injury in $34 \%$ (31/92), relating to inadequate pedicle width. Of the patients, only $11 \%$ $(5 / 46)$ were deemed to have low risk of vertebral artery injury during placement of long pedicle screws bilaterally, whereas $15 \%(7 / 46)$ were at high risk of vertebral artery injury bilaterally and $20 \%$ (9/46) were at moderate risk of injury bilaterally. The remaining 54\% (25/46) showed variable pedicle width between the left and right sides, with 17 of these patients having a high risk of vertebral artery injury on one side (Table 1). 
Table 2. Type of instrumentation placed.

\begin{tabular}{lcccc}
\hline & Pedicle & Pars & Lamina & No Screw \\
\hline Low risk, n & 18 & 1 & 1 & \\
Moderate risk, n & 21 & 3 & & \\
High risk, n & 5 & 6 & 1 & 4 \\
\hline
\end{tabular}

The designation of pedicles as low, moderate, and high risk on the basis of sagittal CT scan slices was reliable. Intraobserver reliability correlation coefficients were excellent at .924 and .914 for the left and right pedicles, respectively. The interobserver reliability correlation coefficients were good at .682 and .658 , respectively. This indicated agreement among reviewers regarding the space available for pedicle screw placement.

A total of 60 pedicles were evaluated in the clinical portion of this study. Risk stratification was determined on the basis of the pedicle width, determined by the number of sagittal slices between the medial wall cut and the vertebral artery cut. Twenty (33\%) pedicles were considered low risk, 24 $(40 \%)$ moderate risk, and $16(27 \%)$ high risk for screw placement. Prior to this study, the actual decision to proceed with pedicle screw placement was a clinical judgment call based upon evaluation of the pedicle's morphology, its relative height on the sagittal view (which was required to be $\geq 3.5$ $\mathrm{mm}$ ), and its width based upon sagittal CT scan slices. As such, pedicle screw placement was deemed feasible and therefore was attempted in 19 of 20 $(95 \%)$ low-risk pedicles and 21 of 24 (88\%) moderate-risk pedicles compared with 5 of 16 (31\%) high-risk pedicles $(P<.001$ comparing low and moderate-risk with high-risk pedicles). Pedicle screws were successfully placed in all but one patient with a low-risk pedicle. In this patient, a breach in the medial wall was noted intraoperatively prior to placing a pedicle screw, thus necessitating pars screw placement. This was the only unsuccessfully attempted pedicle screw placement.

Pedicle screw placement was determined to not be feasible and therefore was not attempted in a total of 16 pedicles. This included $2(10 \%)$ patients with a low-risk pedicle, $3(12.5 \%)$ patients with moderaterisk pedicles, and $11(69 \%)$ with high-risk pedicles. Again, the decision to place pedicle screws was based upon aberrant pedicle anatomy, vertebral artery anatomy, and pedicle height less than $3.5 \mathrm{~mm}$ in addition to the risk assessment based upon sagittal CT scan slices. Pars screws, laminar screws, or no fixation was placed in these cases on the basis of the clinical judgment of the senior surgeon intraoperatively. A summary of screw placement based upon the risk of vertebral artery injury can be found in Table 2.

Postoperative CT scans were available in 22 patients $(73 \%)$. Twenty-nine pedicle screws were placed, of which $28(97 \%)$ were located entirely within the bony corridor. Only 1 pedicle screw placed in a high-risk pedicle demonstrated a medial cortical wall breach, which measured less than 2 $\mathrm{mm}$. In addition, 10 pars or laminar screws were placed. This resulted in 2 additional cortical breaches, including 1 medial wall breach and 1 posterior foraminal breach. Each measured less than $2 \mathrm{~mm}$. No vertebral artery injuries, loss of neurological function or cerebrovascular accidents were observed.

\section{DISCUSSION}

This study presents a simple technique for assessing the complex anatomy of the $\mathrm{C} 2$ pedicle on sagittal CT imaging to allow for safe pedicle screw placement. The excellent intraobserver reliability scores further indicate its reliability, and the clinical data demonstrate the efficacy and safety of this technique in determining the feasibility of screw placement with appropriate length and trajectory.

Feasibility of pedicle screw placement correlates with the risk of vertebral artery injury because these pedicles are inherently smaller and the risk of cortical breach becomes higher. A long $(>22 \mathrm{~mm})$ screw with a diameter from 3.5 to $4.0 \mathrm{~mm}$ can usually be placed entirely within the pedicles of patients with 3 or more cuts showing continuous bone. However, patients with at least 2 cuts showing a pedicle width of approximately $4 \mathrm{~mm}(63 \%$ in this study) might be candidates for the placement of a long screw if a more meticulous technique is used. For example, the drill and screw could be angulated in a cephalad and medial trajectory to avoid the vertebral artery foramen, and different screw lengths and diameters (eg, $3.5 \mathrm{~mm}$ ) could be used, depending on the CT findings. This more meticulous technique was applied in all patients with moderaterisk pedicles in which pedicle screw placement was deemed feasible (ie, height $\geq 3.5 \mathrm{~mm}$ ). Likewise, a properly directed screw might also be safely placed if there are more than $3.5 \mathrm{~mm}$ of bone above the semicircle even in patients with high-risk pedicles. Patients with high-risk pedicles have an inherently "high-riding" vertebral artery. A more cephalad 
starting point can allow placement of a pedicle screw in these patients, as demonstrated in the clinical portion of this study; however, the majority of high-risk pedicles should not be instrumented with a long pedicle screw.

In these patients, the high-riding position of the vertebral artery relative to the pedicle typically prohibits placement of a pedicle screw, as seen in this study; however, a shorter $(<16 \mathrm{~mm})$ pars screw or laminar screw can usually be placed safely. The appropriate length pars screw can be determined by measuring the distance from the posterior cortex of the lamina to the posterior aspect of the semicircle. Likewise, the feasibility and appropriate length laminar screw can be determined from axial $\mathrm{C} 2$ cuts. This trend was seen clinically because these alternative fixation methods were used more frequently in patients with smaller pedicles at high risk for vertebral artery injury.

In the clinical portion of this study, we confirmed our hypothesis that pedicle screws can be safely placed in the majority of patients with low- and moderate-risk pedicles. This was the case in approximately $90 \%$ of these cases, whereas pedicle screws were able to be placed in fewer than onethird of patients with high-risk pedicles. The majority of these latter patients had either no screw or a pars or laminar screw placed.

Three unexpected findings were encountered: (1) only $28 \%$ of the overall number of pedicles had a safe zone of at least $4 \mathrm{~mm}$ through the pedicle; (2) there was high variability $(54 \%)$ in the shape and size of patients' left and right C2 pedicles; and (3) only $11 \%$ of patients had a safe zone $\geq 4 \mathrm{~mm}$ bilaterally.

Resnick et $\mathrm{al}^{6}$ and Yoshida et $\mathrm{al}^{11}$ also examined the radiographic anatomy of $\mathrm{C} 2$ for pedicle screw placement and concluded that pedicle screw placement was feasible in $91 \%$ and $90 \%$ of cases, respectively. In these studies they used sophisticated CT templating software for determination. Unlike the current study, no clinical data were presented for direct comparison, which limits the strength of these conclusions. In addition, these authors' findings are in contrast with the finding that $34 \%$ of pedicles were at high risk for vertebral artery injury during placement of a long pedicle screw and that $52 \%$ of patients had at least one high-riding vertebral artery. Furthermore, the significant variability $(54 \%)$ in the bony anatomy between patients' left and right pedicles noted in the current study was not mentioned in either article. Pruthi et $\mathrm{al}^{14}$ noted similar side-to-side differences in a CT-based study of $\mathrm{C} 2$ pedicles.

In the clinical portion of this study, 44 of 60 $(73 \%)$ pedicles were instrumented with pedicle screws, which, when compared with the aforementioned studies, may indicate that the use of sophisticated CT templating software may allow for a higher percentage of patients to undergo $\mathrm{C} 2$ pedicle screw placement instead of the described technique using standard CT scans and intraoperative fluoroscopy. However, the aforementioned differences in patient anatomy may account for these findings as well. Nevertheless, 56 of 60 (93\%) patients in the current clinical study had some form of fixation placed within the pedicle, pars or lamina using readily available CT scans. Furthermore, asymptomatic cortical breach into the spinal canal or vertebral artery foramen was detected in 1 of 32 (3\%) patients with low- or moderate-risk pedicles and in 2 of $7(29 \%)$ with high-risk pedicles, indicating low risk for this complication. Nonetheless, a potential weakness of this technique is its reliance on 2-dimensional images to gauge the size of a 3-dimensional structure. This inherently allows for error in determining the exact size of the pedicle. Angulated sagittal reconstruction views that parallel the axis of the pedicle are likely to more accurately show the width of the pedicles compared with the method described in this study; however, this capability may not be available at most institutions.

The use of sophisticated CT templating software may decrease the incidence of cortical violation compared with the current technique; however, no clinically significant complications, such as vertebral artery injuries, loss of neurological function, or cerebrovascular accidents were observed. Furthermore, the described technique does not offer a holistic assessment of risk. In the clinical portion of the study, aberrant pedicle morphology and pedicle height were also considered prior to $\mathrm{C} 2$ pedicle screw placement. Although the current technique offers a "quick and easy" assessment of risk, it does not objectively assess specific pedicle morphology, instead relying on the clinical experience of the surgeon to make this determination. Therefore, future prospective study categorizing pedicle morphology and its associated risk profile with $\mathrm{C} 2$ pedicle screw placement is warranted. 


\section{CONCLUSION}

This study describes a technique with excellent individual reproducibility to accurately assess the safety and feasibility of placing a $\mathrm{C} 2$ pedicle screw preoperatively, using tools readily available to essentially all spine surgeons. It is likely more conservative and less complicated than other techniques that have been described; however, as with any technique, it must be used as a tool with the final decision of whether to place a pedicle screw relying on the judgment of the individual surgeon.

\section{REFERENCES}

1. Menendez JA, Wright NM. Techniques of posterior C1C2 stabilization. Neurosurgery. 2007;60(1 supp1 1):S103-S111.

2. Hostin RA, Wu C, Perra JH, Polly DW, Akeson B, Wroblewski JM. A biomechanical evaluation of three revision screw strategies for failed lateral mass fixation. Spine. 2008;33(22):2415-2421.

3. Rhee JM, Kraiwattanapong C, Hutton WC. A comparison of pedicle and lateral mass screw construct stiffnesses at the cervicothoracic junction: a biomechanical study. Spine. 2005;30(21):E636-E640.

4. Ma XY, Yin QS, Wu ZH, et al. $\mathrm{C} 1$ pedicle screws versus $\mathrm{C} 1$ lateral mass screws: comparisons of pullout strengths and biomechanical stabilities. Spine. 2009;34(4):371-377.

5. Harms J, Melcher RP. Posterior C1-C2 fusion with polyaxial screw and rod fixation. Spine. 2001;26:2467-2471.

6. Resnick DK, Lapsiwala S, Trost GR. Anatomic suitability of the C1-C2 complex for pedicle screw fixation. Spine. 2002;27(14):1494-1498.

7. Madawi AA, Casey ATH, Solanki GA, Tuite G, Veres $\mathrm{R}$, Crockard HA. Radiological and anatomical evaluation of the atlantoaxial transarticular screw fixation technique. $J$ Neurosurg. 1997;86(6):961-968.

8. Madawi AA, Solanki G, Casey ATH, Crockard HA. Variation of the groove in the axis vertebra for the vertebral artery. Implications for instrumentation. J Bone Joint Surg Br. 1997;79(5):820-823.

9. Paramore CG, Dickman CA, Sonntag VK. The ana- tomical suitability of the C1-2 complex for transarticular screw fixation. J Neurosurg. 1996;85(2):221-224.

10. Mandel IM, Kambach BJ, Petersilge CA, Johnstone B, Yoo JU. Morphologic considerations of C2 isthmus dimensions for the placement of transarticular screws. Spine. 2000;25(12):1542-1547.

11. Yoshida M, Neo M, Fujibayashi S, Nakamura $T$. Comparison of the anatomical risk for vertebral artery injury associated with the C2-pedicle screw and atlantoaxial transarticular screw. Spine. 2006;31(15):E513-E517.

12. Solanki GA, Crockard HA. Preoperative determination of safe superior transarticular screw trajectory through the lateral mass. Spine. 1999;24(14):1477-1482.

13. Fleiss JL, Cohen J. The equivalence of weighted kappa and the intraclass correlation coefficient as measures of reliability. Educ Psychol Meas. 1973;33:613-619.

14. Pruthi N, Dawn R, Ravindranath Y, Maiti TK, Ravindranath R, Philip M. Computed tomography-based classification of axis vertebra: choice of screw placement. Eur Spine J. 2014;23(5):1084-1091.

Disclosures and COI: This study was performed with Institutional Review Board approval. Dr. Marco is on the speakers bureau for Depuy Synthes and Globus. For the remaining authors, none were declared.

Corresponding Author: Rex A.W. Marco, MD, Houston Methodist Hospital, Department of Orthopaedic Surgery, 6445 Fannin Street, Outpatient Center, Suite 2500, Houston, TX 77030. Phone: (713) 363-7510; Fax: (713) 790-6202; Email: rexmarco@gmail.com.

Published 30 March 2018

This manuscript is generously published free of charge by ISASS, the International Society for the Advancement of Spine Surgery. Copyright (C) 2018 ISASS. To see more or order reprints or permissions, see http://ijssurgery.com. 